\title{
ESTRESSE E COMPORTAMENTO DE COLABORAÇÃO EM FACE DO TRATAMENTO ODONTOPEDIÁTRICO'
}

Cármen Lúcia Cardoso* Sonia Regina Loureiro"

\begin{abstract}
RESUMO. A pesquisa foi realizada no contexto da disciplina de odontopediatria em uma clínica-escola. Objetivou-se caracterizar os problemas comportamentais das crianças atendidas e as manifestações de estresse das crianças, dos seus acompanhantes e dos alunos de odontologia, além de identificar o medo diante do tratamento odontológico dos acompanhantes e as possíveis associações entre as manifestações de estresse e a colaboração das crianças em face dos procedimentos odontológicos. Participaram do estudo 110 crianças, 110 acompanhantes e 70 alunos. Procedeu-se à aplicação dos seguintes instrumentos: Escala de Estresse Infantil; Escala Comportamental A2 de Rutter; Inventário de Sintomas de Estresse de Lipp; Dental Fear Survey. Constatou-se que o atendimento odontopediátrico mostrou-se permeado de manifestações de estresse por parte das crianças, acompanhantes e alunos, condição pouco favorecedora da aprendizagem dos alunos e de comportamentos de colaboração com o atendimento por parte das crianças, o que se fez presente em procedimentos de diferentes níveis de invasão.
\end{abstract}

Palavras-chave: estresse, ansiedade, tratamento odontopediátrico.

\section{STRESS AND COLLABORATION BEHAVIOR IN FACING PEDIATRIC DENTAL TREATMENT}

\begin{abstract}
Current study, carried out at the Pediatric Dentistry Clinic, characterizes behavioral problems and stress manifestations in children undergoing dental treatment and identifies possible links between children's cooperation in dental procedures. Manifestations of stress in the accompanying person and in the dental student practicing the treatment were identified, showing the manner they influence the child behavior during the procedure. One hundred and ten children, 110 accompanying persons and 70 students participated in the study. The following instruments were applied: Child Stress Scale, Rutter Parent Scale A2, Lipp Stress Symptoms Inventory, and Dental Fear Survey. Since dental treatment was permeated with stress manifestations by children, accompanying persons and dentistry students, such conditions did not favor the dentistry students' learning or the children's cooperative behavior. Manifestations were present regardless of the invasiveness degree of the procedure.
\end{abstract}

Key words: Stress, anxiety, pediatric dental treatment.

\section{ESTRÉS Y COMPORTAMIENTO DE COLABORACIÓN FRENTE AL TRATAMIENTO ODONTOPEDIÁTRICO}

RESUMEN. La investigación fue realizada en el contexto de la disciplina de odontopediatría en una clínica escuela. Tuvo como objetivo caracterizar los problemas comportamentales de los niños asistidos y las manifestaciones de estrés de los niños, de sus acompañantes y de los alumnos de odontología, además de identificar el miedo ante el tratamiento odontológico de los acompañantes y las posibles asociaciones entre las manifestaciones de estrés y la colaboración de los niños ante a los procedimientos odontológicos. Participaron del estudio 110 niños, 110 acompañantes y 70 alumnos. Se hizo la aplicación de los siguientes instrumentos: Escala de Estrés Infantil; Escala Comportamental A2 de Rutter; Inventario de Síntomas de Estrés de Lipp; Dental Fear Survey. Se constató que en la asistencia odontopediátrica hubo manifestaciones de estrés, en los niños, acompañantes y alumnos, condición poco favorecedora del aprendizaje de los alumnos y hubo, también, comportamientos de

\footnotetext{
Apoio: FAPESP.
}

* Doutora em Psicologia. Professora do Departamento de Psicologia e Educação da Faculdade de Filosofia, Ciências e Letras de Ribeirão Preto da Universidade de São Paulo.

\# Doutora em Psicologia Clínica. Professora do Departamento de Neurologia, Psiquiatria e Psicologia Médica da Faculdade de Medicina de Ribeirão Preto da Universidade de São Paulo. 
colaboración con la asistencia por parte de los niños, lo que se hizo presente en procedimientos de diferentes niveles de invasión.

Palabras-clave: estrés, ansiedad, tratamiento odontopediátrico.

As faculdades de Odontologia e seus currículos enfatizavam a aquisição de habilidades técnicas e de precisão, sendo recente o reconhecimento de que os aspectos comportamentais e emocionais dos pacientes e dos dentistas são essenciais para o resultado final do tratamento odontológico (Eli, 1992). Essa autora enfatiza que a situação de tratamento odontológico está profundamente relacionada com manifestações de ansiedade e estresse, uma vez que existem estímulos fisiológicos - como, por exemplo, a dor - e aspectos psicológicos envolvidos no tratamento dentário que podem ser avaliados pelos pacientes como potencialmente ameaçadores ao seu bem-estar.

Jacob (1998) afirma que o estresse vivenciado pelo paciente amplia as percepções de medo e de dor, diminuindo a sua capacidade de colaborar com o tratamento.

As manifestações de estresse se fazem presentes em diferentes situações e contextos. O primeiro autor a utilizar o termo estresse foi Selye (1956), observando que pacientes com diferentes síndromes apresentavam um conjunto de reações emocionais não específicas. A reação de estresse foi definida por Selye (1956) como uma síndrome geral de adaptação (SGA), na qual a principal ação do estresse seria a quebra da homeostase interna. Para a compreensão da evolução das alterações físicas e químicas produzidas pelo estresse, Selye (1956) apresentou um modelo no qual diferencia três fases, a saber: de alerta, de resistência e de exaustão.

Crianças e adultos estão suscetíveis ao estresse, e considera-se que a vivência de um estresse está associada a consequiências negativas para a vida das pessoas.

Em relação à atenção odontológica, o estresse e a ansiedade têm sido relacionados com a evitação ou fuga do tratamento, representando uma barreira à utilização desses serviços, mesmo quando muito necessários, comprometendo a saúde bucal do indivíduo (Cesar, Moraes, Milgrom \& Kleinknecht, 1993; Moraes, Ambrosano \& Possobon, 2004).

Segundo observa Giron (1988), os pacientes pediátricos não têm escolha e são geralmente levados pelos pais para o tratamento dentário. Tais pacientes em geral manifestam o seu medo através de seus comportamentos como, por exemplo, choro, recusa em abrir a boca, chute, na tentativa de evitar o atendimento odontológico.
De modo geral a ansiedade diante do tratamento dentário tem sido relacionada a uma etiologia multifatorial, influenciada principalmente por aspectos internos ao indivíduo, como o ambiente no qual ele vive e ainda a própria situação de atendimento odontológico (Corkey \& Freeman, 1994; Eli, Uziel, Bath \& Kleinhauz, 1997).

Entre estes fatores a importância da relação com a mãe é ressaltada por Corkey e Freeman (1994) pelo seu duplo papel; ou seja, tal relação influencia o desenvolvimento psicológico da criança e as suas habilidades para enfrentar a situação odontológica. Possobon, Caetano e Moraes (1998) ressaltam a importância das primeiras relações com o dentista. Estes autores descrevem que "as experiências odontológicas iniciais deveriam ocorrer com um mínimo de trauma físico e psicológico" (p. 80).

Especificamente em relação aos profissionais da área de odontologia considera-se que a prática nesta profissão está associada com altos níveis de estresse (Newton et al., 1994). Jacob (1998) afirma que o cirurgião-dentista, quando submetido ao estresse, diminui a sua tolerância às diferentes situações que terá que enfrentar.

O aluno de odontologia, tal qual o profissional, está submetido a vários fatores que são potencialmente estressantes. Diversos estudos relacionam os níveis de estresse vivenciados pelos alunos com uma diminuição no seu desempenho durante a graduação (Macchi, Biondi \& Cortese, 1996; Newton et al., 1994; Sturdevant, George \& Lundeen, 1987; Tedesco, 1986).

Tedesco (1986) afirma que altos níveis de estresse associam-se a um aumento de sintomas físicos e psicológicos, como dor de cabeça, dor nas costas, insônia, hostilidade, depressão e raiva, incidindo negativamente na qualidade da aprendizagem e na execução de trabalhos dos alunos, independentemente de suas habilidades cognitivas e técnicas.

De modo geral os alunos enfrentam as demandas da realização dos primeiros trabalhos clínicos no contexto de clínicas-escola realizando procedimentos odontológicos sob orientação e supervisão de docentes, que por sua vez avaliam os alunos por meio destas atividades. Segundo Klatchoian (1993), nas instituições os atendimentos odontológicos tendem a supervalorizar a atuação e a produtividade do profissional, sendo pouco relevantes os sentimentos 
das crianças e do próprio cirurgião-dentista, o que por sua vez facilita o uso de atitudes e práticas autoritárias. A relação entre dentista e paciente é mais impessoal, ou seja, a individualidade do paciente é relegada a segundo plano, uma vez que o vínculo principal é aquele entre o paciente e a instituição, sendo muitas vezes priorizadas as regras da instituição.

Com base em estudo conduzido em clínica-escola, envolvendo crianças com altos níveis de ansiedade diante do tratamento odontológico, Cardoso e Loureiro (2005) apontam que tal situação constitui-se em um campo propício para o desenvolvimento de trabalhos conjuntos entre a Psicologia e a Odontologia sob a perspectiva de um modelo integrado de atenção à saúde. Destacam ainda que, neste contexto, a interface Psicologia e Odontologia pode se efetivar no diagnóstico, no planejamento e no atendimento a crianças.

Considerando que no contexto de clínica-escola o atendimento odontopediátrico envolve demandas que, pelas suas peculiaridades, configuram a participação de criança, acompanhante e aluno, objetiva-se avaliar as manifestações de estresse que permeiam tal situação, buscando identificar as possíveis associações com os procedimentos odontológicos e a colaboração das crianças em face desses procedimentos.

Constituem objetivos específicos: a) caracterizar o perfil comportamental e as manifestações de estresse de crianças em idade escolar, atendidas em uma clínica-escola de odontopediatria; b) caracterizar as manifestações de estresse e de medo odontológico dos acompanhantes; c) caracterizar as manifestações de estresse dos alunos de odontologia.

A hipótese central que agrupa tais objetivos pressupõe que as manifestações emocionais dos diferentes atores dificultam a colaboração da criança com os procedimentos odontológicos.

\section{MÉTODO}

O estudo foi desenvolvido na Clínica de Odontopediatria da Faculdade de Odontologia de Ribeirão Preto (FORP/USP), nas condições naturais de atendimento da clínica, após aprovação pelo Comitê de Ética em Pesquisa (Processo no 101299) e assinatura do Termo de Consentimento Livre e Esclarecido por parte dos participantes.

\section{Participantes}

Crianças - A amostra foi composta de 110 crianças atendidas na Clínica de Odontopediatria, o que corresponde a $86.6 \%$ do total de 127 crianças atendidas, nesta faixa etária, no período de um ano, após a aplicação dos critérios de exclusão, a saber: crianças com má-formação congênita, com problemas neurológicos e/ou psicológicos já diagnosticados ou tratados, com internações hospitalares por problemas de saúde e não residentes com a família biológica.

Observou-se, em relação à caracterização das crianças, predominância de participantes do sexo feminino $(58,2 \%)$ sobre participantes do sexo masculino (41,8\%). Quanto a variável idade, com variação de 6 a 12, observou-se um predomínio percentual de participantes com idades de 6 anos a 7 anos e 11 meses $(50,0 \%)$. A escolaridade das crianças variou de pré-escola à $6^{\mathrm{a}}$ série, e o maior percentual de crianças estava na pré-escola e primeira série do ensino fundamental $(46,3 \%)$.

Acompanhantes - Dos 110 acompanhantes das crianças, $91,8 \%$ foram mães. Em relação à escolaridade, a maioria das mães $(80,0 \%)$ possuía entre $1^{\mathrm{a}}$ e $8^{\mathrm{a}}$ séries do ensino fundamental. A renda familiar dos participantes variou de sem renda $(3,6 \%)$ até 12 salários-mínimos $(0,9 \%)$, com predomínio na faixa de 1 a 2 salários-mínimos $(56,4 \%)$.

Alunos - Foram avaliados 70 alunos que realizavam o atendimento das crianças. Os alunos cursavam o $4^{\circ}$ ano do curso de Odontologia da Faculdade de Odontologia de Ribeirão Preto / USP. A média de idade foi de 21 anos, com variação de 20 a 26 anos. Em relação ao gênero, $45,7 \%$ eram do sexo feminino e $54,3 \%$ do masculino.

\section{Instrumentos}

- Escala de Stress Infantil (ESI) - utilizada para a avaliação do estresse infantil e do tipo de reações mais frequiente, ou seja: física, psicológica, psicológiaca com componente depressivo e psicofisiológica. Contém 35 itens em uma escala do tipo likert de cinco pontos. Foi desenvolvida e validada por Lipp e Lucarelli (1998) e a análise fatorial evidenciou os quatros fatores acima descritos e um fator geral: estresse. Refere bons indicadores de consistência interna (coeficiente alfa de Cronbach 0,90).

- Escala Comportamental Infantil A2 de Rutter (ECI) - utilizada para a avaliação de problemas comportamentais e emocionais das crianças, adaptada e padronizada para o nosso meio por Graminha (1994). Contém 36 itens distribuídos em três tópicos, que são: problemas de saúde (oito itens), hábitos (sete itens) e comportamento (21 itens). O respondente deve assinalar a freqüência ou a intensidade do problema apresentado. A nota 
de corte maior que 16 foi considerada indicativa de problemas comportamentais, requerendo ajuda especializada. Possui índices psicométricos adequados, com valores de fidedgnidade (teste e reteste) aceitáveis para $97 \%$ dos itens da escala adaptada e bons indicadores de validade discriminante na identificação de casos que necessitam de ajuda psicológica.

- Dental Fear Survey (DFS) - utilizada para a avaliação da intensidade do medo ante a situação de tratamento odontológico. Foi validado para o Brasil por Cesar et al. (1993). O instrumento contém 20 itens em uma escala do tipo likert de cinco pontos, na qual o sujeito deve assinalar a freqüência ou a intensidade com que avalia experimentar a situação relatada. Possui índices psicométricos adequados e bons indicadores de validade discriminante na identificação de casos com alto medo odontológico.

- Inventário de Sintomas de Stress para Adultos de Lipp (ISSL) - validado por Lipp (2000). É utilizado para a avaliação de sintomas de estresse, o tipo de sintoma e a fase de estresse em que se encontra, ou seja alerta, resistência, quaseexaustão e exaustão. O Inventário está dividido em três partes, devendo o indivíduo assinalar: na primeira parte os sintomas que apresentou nas últimas 24 horas, numa lista de 15 sintomas de estresse; na segunda parte, os sintomas experimentados na última semana (15 sintomas de estresse); e na terceira parte, o respondente deve assinalar, numa lista de 23 sintomas, os experimentados no último mês. A análise fatorial evidenciou as quatro fases acima descritas e um fator geral - estresse. Refere boa confiabilidade do instrumento com o coeficiente Alfa de Cronbach de 0,91 .

- Ficha de observação - é utilizada para registrar as informações dos alunos relativas ao comportamento de colaboração da criança diante da situação de atendimento. Contém a anotação do procedimento odontológico realizado e uma lista de nove comportamentos durante o tratamento dentário, a saber: colaborou com os procedimentos técnicos; dificultou os procedimentos técnicos; precisou ser contida fisicamente; chorou; mordeu; vomitou; queixou-se de dor proporcional à situação; queixou-se de dor desproporcional à situação; e ainda um espaço para anotações de observações relativas a situações específicas. Tal ficha foi preenchida pelos alunos imediatamente após cada sessão de atendimento das crianças. A pontuação de uma criança numa sessão de tratamento poderia variar de 0 a 28 , sendo que 0 representa o máximo possível de respostas de colaboração e 28 o máximo possível de respostas de não-colaboração. O nível de invasão dos procedimentos a que as crianças foram submetidas foi objeto de classificação posterior por cinco odontopediatras com experiência na área, a saber: POUCO INVASIVO - exame clínico e radiográfico, avaliação de risco de cárie; profilaxia; aplicação tópica de flúor; polimento de restauração; raspagem supragengival; INVASIVO - dentística (escariação; restauração); aplicação de selante; moldagem; coroa de aço; MUITO INVASIVO - endodontia (pulpotomia, cirurgia de acesso, odontometria, curativo endodôntico) e cirurgia (exodontia e gengivectomia). Os alunos registraram a observação de um total de 816 sessões de atendimento odontológico, o que corresponde a uma média de sete sessões por criança. Os procedimentos de tratamento foram classificados como pouco invasivos em $26,2 \%$ das situações, como invasivos em $48,8 \%$ das situações e como muito invasivos em $25,0 \%$.

\section{Coleta dos dados}

Todas as crianças foram avaliadas quanto ao estresse individualmente, em uma única sessão de aproximadamente 25 minutos. Os acompanhantes avaliaram os comportamentos das crianças com base em uma escala e foram avaliados em termos pessoais quanto ao estresse e ao medo odontológico, individualmente, em situação de entrevista face a face, em uma única sessão com duração média de aproximadamente 40 minutos. A avaliação em termos de estresse dos alunos de odontologia foi realizada coletivamente em sala de aula antecedendo as atividades teóricas da disciplina de odontopediatria. A aplicação do instrumento ocorreu aproximadamente dois meses após o início das atividades de atendimento às crianças, sendo condição que os alunos já tivessem realizado pelo menos duas sessões de tratamento com as crianças.

As técnicas utilizadas foram codificadas de acordo com as proposições técnicas. Os resultados referentes a cada subgrupo de participantes nos instrumentos aplicados foram apresentados em termos de freqüência e porcentagem. Procedeu-se à aplicação do teste $\mathrm{t}$ student para amostras independentes para verificar a associação entre o comportamento de colaboração da criança ante a situação de tratamento 
odontológico e os seguintes instrumentos de avaliação psicológica: ECI, ISSL e o DFS. Foi efetuada análise multivariada (MANOVA) para verificar os efeitos das variáveis, comportamento das crianças obtido na ECI, manifestações de medo e estresse dos acompanhantes sobre o comportamento da criança na situação de tratamento odontológico em diferentes procedimentos odontológicos segundo a avaliação dos alunos. Para verificar a correlação entre a ESI e o comportamento de colaboração das crianças com o atendimento utilizou-se o coeficiente de correlação de Spearman. Para todos os testes estatísticos utilizados adotou-se o nível de significância de $p=0,05$; utilizou-se o programa estatístico Statistc Package for Social Sciences (SPSS) versão 12.0 (Nie \& Hull, 1975).

\section{RESULTADOS}

Os dados referentes ao perfil comportamental e de estresse das crianças, em termos de frequiência e porcentagem, são apresentados na Tabela 1 .

Tabela 1. Perfil das Crianças Relativo ao Comportamento (ECI) e ao Estresse (ESI).

\begin{tabular}{llcc}
\hline Variável & Especificação & F & \% \\
\hline Comportamento & Presença $(>16)$ & 60 & 54,5 \\
(ECI) $(\mathrm{n}=110)$ & Ausência $(\leq 16)$ & 50 & 45,5 \\
Tipo de desordem & Anti social & 35 & 58,3 \\
$(\mathrm{n}=60)$ & Neurótica & 18 & 30,0 \\
& Indiferenciado & 7 & 11,7 \\
Estresse (ESI) & Presença & 23 & 20,9 \\
$(\mathrm{n}=110)$ & Ausência & 87 & 79,1 \\
\hline
\end{tabular}

$\mathrm{F}=$ freqüência $\%=$ Porcentagem

Na tabela 1, que se refere ao comportamento e ao estresse das crianças, verificou-se, com base na avaliação comportamental, tendo-se por referência a nota de corte $(>16)$ para a população brasileira, que $54,5 \%$ das crianças apresentaram resultados acima da nota de corte, sugerindo a presença de indicadores de problemas comportamentais e ainda segundo o acompanhante, a necessidade de encaminhamento para atendimento especializado. Destas crianças observou-se que a maioria $(58,3 \%)$ apresentou indicadores de desordens anti-sociais, indicando uma maior dificuldade de agir de acordo com o socialmente aceito. Quanto à avaliação de estresse constatou-se, com base no estudo normativo, a presença destes indicadores em $20,9 \%$ das crianças.

Os dados relativos aos acompanhantes, em termos de frequiência e porcentagem, são apresentados na Tabela 2.
Tabela 2. Perfil dos Acompanhantes com Relação ao Medo Frente ao Tratamento Odontológico (Dental Fear Survey) e ao Estresse (ISSL).

\begin{tabular}{llcc}
\hline Variáveis & Especificação & F & \% \\
\hline Medo dos & Presença $(\geq 60)$ & 28 & 25,5 \\
Acompanhantes & Ausência $(<60)$ & 82 & 74,5 \\
$(\mathrm{n}=110)$ & & & \\
\hline Estresse do & Presença & 67 & 60,9 \\
Acompanhante & Ausência & 43 & 39,1 \\
(n=110) & & & \\
Presença de Stress & Fase de alerta & 01 & 1,5 \\
(n=67) & Fase de resistência & 53 & 79,1 \\
& Fase de quase exaustão & 11 & 16,4 \\
& Fase de Exaustão & 02 & 3,0 \\
& Sintoma Físico & 7 & 10,4 \\
& Sintoma Psicológico & 55 & 82,1 \\
& Sintoma Físico e Psicológico & 5 & 7,5 \\
\hline
\end{tabular}

$\mathrm{F}=$ freqüência $\%=$ porcentagem

Conforme pode ser observado na Tabela 2, os dados relativos ao medo odontológico dos acompanhantes obtidos através do Questionário Dental Fear Survey, tendo como referência os dados normativos deste instrumento, indicaram a presença de alto medo em face do tratamento odontológico em $25,5 \%$ dos acompanhantes das crianças.

Os resultados relativos às manifestações de estresse dos acompanhantes das crianças foram obtidos através do ISSL e a comparação com os dados normativos indicou a presença de estresse em 60,9\% dos acompanhantes das crianças. Dos acompanhantes que apresentaram manifestações de estresse, a maioria está concentrada na fase de resistência $(79,1 \%)$, com predominância de sintomatologia na área psicológica $(82,1 \%)$.

A Tabela 3 apresenta os resultados relativos aos alunos de odontologia, em termos de freqüência e porcentagem.

Tabela 3. Perfil dos Alunos de Odontologia Quanto ao Stress Obtido Através do ISSL.

\begin{tabular}{llcc} 
Variável & Especificação & F & \% \\
\hline Stress dos Alunos & Presença & 40 & 57,1 \\
(ISSL) (n=70) & Ausência & 30 & 42,9 \\
& & 1 & 2,5 \\
Presença de Stress & Fase de alerta & 35 & 87,5 \\
$(\mathrm{n}=40)$ & Fase de resistência & 3 & 7,5 \\
& Fase de quase exaustão & 1 & 2,5 \\
& Fase de Exaustão & 7 & 17,5 \\
& Sintoma Físico & 33 & 82,5 \\
& Sintoma Psicológico & 0 & 0 \\
\hline
\end{tabular}

$\mathrm{F}=$ freqüência $\%=$ porcentagem

Em relação às manifestações de estresse dos alunos, a Tabela 3 mostra a presença de estresse em 
$57,1 \%$, sendo que estes indicadores se concentram na fase de resistência $(87,5 \%)$, com um predomínio de sintomatologia na área psicológica $(82,5 \%)$.

A Tabela 4 apresenta a comparação entre as médias dos comportamentos de colaboração das crianças em relação ao tratamento odontológico de acordo com a observação dos alunos, em associação com a presença e ausência de dificuldades comportamentais tendo por base a ECI, com indicadores de medo odontológico e as manifestações de estresse dos acompanhantes.

Tabela 4. Comportamento de Colaboração das Crianças Frente aos Procedimentos Odontológicos Associados ao Perfil Comportamental Obtido na ECI, aos Indicadores de Medo Odontológico e de Presença ou Ausência de Estresse dos Acompanhantes.

\begin{tabular}{|c|c|c|c|c|c|c|c|}
\hline \multirow[t]{2}{*}{ Perfil } & \multirow[b]{2}{*}{ Procedimentos } & \multicolumn{2}{|c|}{ Presença } & \multicolumn{2}{|c|}{ Ausência } & \multirow[t]{2}{*}{$\mathbf{t}$} & \multirow[t]{2}{*}{ Comparação } \\
\hline & & M & DP & M & DP & & \\
\hline \multirow[t]{4}{*}{ ECI } & Pouco invasivo & 9,0 & 7,3 & 2,0 & 3,8 & 6,5 & Pres.> Aus.* \\
\hline & Invasivo & 13,9 & 7,1 & 3,7 & 5,7 & 8,2 & Pres.> Aus.* \\
\hline & Muito invasivo & 12,9 & 8,4 & 5,9 & 7,0 & 4,2 & Pres.> Aus.* \\
\hline & Pouco invasivo & 10,2 & 7,6 & 4,3 & 5,9 & 4,3 & Pres.> Aus.* \\
\hline \multirow[t]{3}{*}{ Medo } & Invasivo & 12,9 & 7,9 & 8,0 & 7,9 & 2,8 & Pres.> Aus.** \\
\hline & Muito invasivo & 18,3 & 7,0 & 7,2 & 7,2 & 6,2 & Pres.> Aus.* \\
\hline & Pouco invasivo & 7,9 & 7,5 & 2,4 & 4,0 & 5,0 & Pres.> Aus.* \\
\hline \multirow[t]{2}{*}{ Estresse } & Invasivo & 12,5 & 8,2 & 4,1 & 5,0 & 6,6 & Pres.> Aus.* \\
\hline & Muito invasivo & 13,2 & 8,4 & 5,2 & 6,4 & 5,0 & Pres.> Aus.* \\
\hline
\end{tabular}

Teste $\mathrm{t}$ para amostras independentes $* * \mathrm{p}<0,01 * \mathrm{p}<0,001 \quad \mathrm{M}=$ Média DP=Desvio Padrão

O comportamento de colaboração foi avaliado com base no registro efetuado pelos alunos que realizavam o tratamento odontológico. Quanto maior o valor atingido, menor o comportamento de colaboração com a situação de atendimento.

De acordo com a Tabela 4, observa-se que em todos os níveis técnicos de procedimento as crianças com indicadores de presença de problemas de comportamento (escore > 16 - ECI) apresentaram médias significativamente maiores que as crianças com indicadores de ausência de problemas de comportamento (escore $\leq 16-\mathrm{ECI}$ ), indicando que as crianças com maiores dificuldades comportamentais e emocionais de acordo com a avaliação dos acompanhantes, apresentaram também menos colaboração ante a situação de tratamento odontopediátrico, segundo a avaliação dos alunos. Em relação aos acompanhantes, pôde-se verificar que as crianças cujos acompanhantes apresentaram indicadores de elevado medo diante do tratamento odontológico apresentaram mais dificuldades em colaborar com os procedimentos de tratamento odontológico quando comparadas às crianças cujos acompanhantes apresentaram ausência de indicadores de medo odontológico. As crianças cujos acompanhantes apresentaram indicadores de presença de estresse obtiveram médias significativamente maiores em todos os níveis de procedimentos odontológicos, quando comparadas às crianças cujos acompanhantes não apresentaram indicadores de estresse, sugerindo associação de menor colaboração com a situação de tratamento odontológico e a presença de estresse por parte acompanhante.

A análise de correlação de Spearman entre os escores obtidos de estresse das crianças e os comportamentos de colaboração destas mostra uma correlação positiva entre os escores totais na Escala de Stress Infantil e as dificuldades diante do atendimento odontológico, ou seja, conforme aumentaram as manifestações de estresse das crianças, aumentaram significativamente as dificuldades de colaboração com o tratamento odontológico nos procedimentos classificados como invasivos $(r=0,23 p<0,02)$ e muito invasivos $(r=0,23 p=0,03)$. Vale destacar ainda que a correlação para os procedimentos avaliados como pouco invasivos mostrou-se fronteiriça, apontando para a mesma tendência $(r=0,18 p=0,06)$.

Através da análise multivariada para os comportamentos de colaboração das crianças com o tratamento odontológico em cada procedimento, tendo como variáveis independentes o perfil comportamental da criança obtido na ECI, o medo e o estresse do acompanhante, verificou-se que as três variáveis independentes agem significativamente sobre a variável comportamento da criança avaliado pelo dentista no procedimento pouco invasivo $(\mathrm{ECI} \rightarrow$ $F=25,7 p=0,000 ;$ Medo $\rightarrow \mathrm{F}=7,2 p=0,008$; Estresse $\rightarrow$ $F=5,0 p=0,027)$. Observou-se, ainda que as variáveis independentes perfil comportamental das crianças obtido na ECI $(F=48,5 p=0,000)$ e estresse dos acompanhantes $\quad(F=18,7 \quad p=0,000) \quad$ agem significativamente sobre a variável comportamento da criança avaliado pelo dentista no procedimento invasivo; já a variável medo do acompanhante $(F=0,4$ $p=0,533)$ não age significativamente neste nível de procedimento. Finalmente, em relação ao comportamento das crianças avaliado pelo dentista no procedimento muito invasivo, observou-se que as três variáveis independentes $(\mathrm{ECI} \rightarrow F=8,5 \quad p=0,005$; Medo $\rightarrow F=20,1 p=0,000 ;$ Estresse $\rightarrow F=5,1 p=0,026$ ) agem significativamente sobre esse comportamento.

\section{DISCUSSÃO}

Faz-se necessário destacar inicialmente, na descrição do perfil socioeconômico das crianças e acompanhantes atendidas na clínica-escola, o baixo 
nível de renda e escolaridade desta população, quando comparado aos dados do Instituto Brasileiro de Geografia e Estatística (IBGE, 2000). Na cidade de Ribeirão Preto $31,04 \%$ da população recebem remuneração de até dois salários mínimos e na amostra que frequienta a clínica-escola $56,4 \%$ estão nesta faixa salarial. Em relação à escolaridade, $80 \%$ dos acompanhantes das crianças possuem escolaridade até o ensino fundamental, já para a população de Ribeirão Preto 47,5\% possuem este nível de escolaridade. Embora este dado de certa forma fosse esperado, é possível refletir sobre como se dá o estabelecimento de relações entre aluno, criança e acompanhante (IBGE, 2000).

Com relação ao perfil das crianças, verificou-se que a maioria destas apresentou dificuldades comportamentais e emocionais, indicando a necessidade de acompanhamento psicológico, segundo os acompanhantes. Estas dificuldades, pelas características dos itens pesquisados, são anteriores ao tratamento odontológico, mas parecem influenciar a situação de atendimento, uma vez que estas crianças, também na percepção dos alunos, apresentaram mais dificuldades para a realização do tratamento.

Para a maioria destas crianças a problemática é de natureza anti-social, caracterizando dificuldades externalizadas no meio de forma direta pela agressividade e oposição, apontando para um padrão comportamental pouco socializado e adaptado.

A situação de tratamento odontológico tem sido descrita como potencialmente estressante (Eli, 1992), podendo desencadear reações de estresse em pacientes vulneráveis. Pode-se pensar que as crianças com dificuldades emocionais e comportamentais se encontram nesta condição, e ao experimentarem um aumento da ansiedade diante do tratamento odontológico, podem expressar comportamentos pouco colaboradores com a situação de atendimento odontológico, como constatado no estudo.

Observou-se ainda que $20,9 \%$ das crianças apresentaram indicadores de manifestações de estresse, com correlação entre a presença de estresse e maior dificuldade diante do tratamento odontológico, na percepção do aluno. Ao analisar os dados relativos ao comportamento, estresse e colaboração das crianças com o tratamento odontológico, destaca-se a concordância destes com a afirmativa de Lipp, Souza, Romano e Covolan (1991) quanto a poderem as manifestações de comportamentos agressivos ser formas utilizadas pelas crianças para o alívio e redução da ansiedade, minimizando o estresse do momento, caracterizando assim um comportamento reforçado pelas conseqüências que produz.
Outro ponto a ser discutido diz respeito aos indicadores de estresse apresentados pelas acompanhantes - mães das crianças. Observou-se que a maioria dos acompanhantes (mães) apresentou indicadores de manifestações de estresse, de acordo com os critérios normativos propostos por Lipp (2000). Com relação à fase de predomínio do estresse, identificou-se a fase de resistência, o que caracteriza a permanência do estresse, seja pela intensidade do estressor, por se manter por muito tempo, seja por dificuldade do sujeito para enfrentar as situações estressantes. Segundo Selye (1983), na fase de resistência o indivíduo está mais enfraquecido e mais susceptível a doenças, ocorrendo um dispêndio de energia adaptativa, porque o organismo tenta restabelecer o equilíbrio interno através de uma ação reparadora.

Nos dados do presente estudo observou-se que os indicadores de estresse apresentados pelos acompanhantes - em geral, mães das crianças - são principalmente de natureza psicológica. Ao tentar compreender este dado, pode-se pensar que estas mães, vivenciando estresse com sintomatologia psicológica, encontram-se mais ansiosas, irritadas, com hipersensibilidade emotiva, pouco confiantes em si mesmas e ainda com menor capacidade para acolher as dificuldades apresentadas pelos seus filhos, dada a presença dos indicadores de problemas comportamentais e de estresse.

Ao se analisar a associação entre a presença de indicadores de estresse do acompanhante e a manifestação de dificuldade de colaboração por parte das crianças, segundo a avaliação dos alunos, pode-se pensar que a menor tolerância por parte dos acompanhantes - em 91,8\% dos casos, mães - possa estar relacionada à presença de indicadores de sobrecarga que podem estar minimizando suas condições para acolher as demandas das crianças e ajudá-las a enfrentar o estresse da situação de tratamento odontológico.

Os estudos desenvolvidos por Corkey e Freeman (1994) enfatizam a importância da relação com a mãe, uma vez que a forma como a criança tolera o estresse e a habilidade para enfrentá-lo parecem ser facilitadas quando as mães são compreensivas e autoconfiantes e os pais impõem um conjunto de limites. Estes autores afirmam que a relação estabelecida com a mãe influencia as habilidades da criança para enfrentar a situação odontológica.

Os dados acerca do medo odontológico mostraram que $25,5 \%$ dos acompanhantes-mães apresentaram indicadores de alto medo diante do tratamento. A presença de indicadores de medo nos acompanhantes- 
mães foi associada a maiores dificuldades de colaboração por parte das crianças com o tratamento odontológico, de acordo com avaliação dos alunos. Supõe-se assim que os sentimentos e as atitudes dos acompanhantes - em geral, mães - em face do tratamento odontológico possam influenciar a percepção da criança ante a situação de atendimento. Neste sentido, destaca-se o papel dos acompanhantes enquanto modelos para as crianças.

Tal dado é concordante com os de Corkey e Freeman (1994) e Eli et al. (1997), que afirmam que o aparecimento da ansiedade odontológica pode estar relacionado à aprendizagem, na qual a ansiedade é desenvolvida através da observação, da identificação ou sugestão de modelos, geralmente os pais.

Os dados relativos à análise multivariada (MANOVA) demonstraram que as três variáveis comportamento da criança obtido na ECI, estresse e medo dos acompanhantes-mães - estão independentemente associadas ao comportamento da criança na situação de tratamento odontológico avaliado pelo aluno, considerando os procedimentos pouco invasivos e muito invasivos. Em relação ao comportamento das crianças nos procedimentos invasivos, as variáveis comportamento da criança avaliado pelo acompanhante e estresse do acompanhante estão significativamente associadas. A variável medo dos acompanhantes não chegou a exercer influência significativa.

$\mathrm{O}$ risco de a criança apresentar problemas comportamentais na situação de tratamento odontológico é maior nas crianças que apresentam problemas comportamentais segundo a avaliação do acompanhante e também naquelas cujos acompanhantes apresentam manifestações de estresse e alto medo odontológico.

Os dados relativos aos indicadores de estresse apresentados pelos alunos de odontologia apontaram que a maioria deles, de forma semelhante aos acompanhantes/mães, apresentou manifestações de estresse $(57,1 \%)$ principalmente na fase de resistência e com predomínio de sintomatologia psicológica.

Este achado é concordante com outros estudos (Newton et al., 1994; Sturdevant et al., 1987; Tedesco, 1986), que afirmaram que o aluno de odontologia está submetido a vários fatores potencialmente estressantes.

Segundo Tedesco (1986), os níveis de estresse vivenciados pelos alunos influenciam negativamente a qualidade da aprendizagem e a execução de trabalhos, independentemente das habilidades cognitivas e técnicas destes alunos. Essa autora afirma que o estresse está associado a um aumento de sintomas físicos e psicológicos, como dor de cabeça, dor nas costas, hostilidade, depressão, raiva e outros.

A condição emocional e física do cirurgião dentista tem sido destacada como um elemento importante na situação de tratamento odontopediátrico, uma vez que ela afeta a percepção que a criança tem desta situação (Alwin, Murray \& Niven, 1994).

Constatou-se que, no contexto da clínica-escola, o atendimento odontopediátrico relatado no estudo em questão mostrou o predomínio de manifestações de estresse por parte das crianças, acompanhantes e alunos, condição pouco favorecedora da aprendizagem dos alunos e de comportamentos de colaboração com o atendimento por parte das crianças, o que se manifestou em procedimentos de diferentes níveis de invasão. Destaca-se aqui que tais características associadas às manifestações de estresse e comportamentais das crianças parecem se sobrepor à situação em si, na medida que, mesmo diante de procedimentos pouco invasivos, relacionados à baixa possibilidade de dor, observou-se pouca colaboração das crianças. Confirmou-se assim a hipótese formulada relativa à interferência negativa das manifestações emocionais dos diferentes atores no comportamento de colaboração das crianças diante do tratamento odontológico, o que por sua vez acrescenta demandas à situação de aprendizagem por parte dos alunos.

Neste contexto, destaca-se a necessidade de estruturar intervenções que possam instrumentar os alunos a enfrentarem melhor o início dos trabalhos clínicos em odontopediatria, favorecendo o desenvolvimento de habilidades para lidar com crianças e acompanhantes que apresentam elevada ansiedade, sob a perspectiva de uma formação multidisciplinar que considere a saúde na sua integralidade. Os estudos conduzidos por Sturdevant et al. (1987) apontaram na mesma direção, ou seja, enfatizaram a necessidade de intervenções que diminuam o estresse vivenciado pelos alunos, capacitando-os a enfrentar melhor as demandas próprias do curso de odontologia.

Em síntese e como considerações finais, detaca-se, com base nos dados relativos à avaliação dos atores envolvidos no atendimento odontopediátrico, no contexto de uma clínica-escola, uma multiplicidade de fatores envolvidos na participação, desde variáveis pessoais e condições mais amplas - como diferenças socioeconômico-culturais - até a situação concreta de demandas próprias dos alunos, acompanhantes e crianças. Ao considerar tais condições se pressupõe uma concepção do processo saúde/doença que vai além do definido no modelo biomédico, com prioridades para execução de procedimentos técnicos independentemente do processo que envolve a sua aplicação.

Pelo fato de o delineamento adotado envolver a coleta de dados no contexto da clínica-escola, sem qualquer interferência na situação de seleção e de 
atendimento, destacam-se os seguintes limites: a ampla faixa etária das crianças incluídas, a restrição dos dados da ficha de observação a um pequeno rol de categorias e a dificuldade de isolar o peso das variáveis que poderiam ser responsáveis pelo comportamento de colaboração das crianças. Neste cenário complexo fazem-se necessários novos estudos que acompanhem o atendimento odontopediátrico ao longo das suas diferentes etapas, ampliando os recursos de observação do processo e das interações para crianças mais jovens e mais velhas.

Ao se considerar o contexto deste estudo sob a perspectiva do modelo biopsicossocial, pressupõe-se o envolvimento de equipes multidisciplinares, com vista a uma maior integralidade das ações de saúde. Neste sentido, a Psicologia, como parceira da Odontologia, poderia colaborar na reflexão e na problematização das intervenções numa postura criativa para a composição dos trabalhos consoantes com as necessidades de todos os atores envolvidos no processo saúde/doença/cuidado. Tal interlocução favoreceria a construção de espaços mais democráticos no trato com a alteridade, promovendo maior integração entre profissionais e usuários e contribuindo para a integralidade da atenção à saúde tão almejada pelo sistema de saúde.

\section{REFERÊNCIAS}

Alwin, N., Murray, J. J. \& Niven, N. (1994). The effect of children's dental anxiety on the behaviour of a dentist. International Journal of Paediatric Dentistry, 4, 19-24.

Cardoso, C. L. \& Loureiro, S. R. (2005). Problemas comportamentais e stress em crianças com ansiedade frente ao tratamento odontológico. Estudos de Psicologia, 22(1), 5-12.

Cesar, J., Moraes, A. B. A., Milgrom, P. \& Kleinknecht, R. A. (1993). Cross validation of a Brazilian version of the Dental Fear Survey. Community Dentistry and Oral Epidemiology, 21(1), 148-50.

Corkey, B. \& Freeman, R. (1994). Predictors of dental anxiety in six year old children: Findings from a pilot study. Journal of Dentistry for Children, 61(4), 267-71.

Eli, I. (1992). Oral Psychophysiology: Stress, pain and behavior in dental care. Florida: CRC.

Eli, L., Uziel, N., Bath, R. \& Kleinhauz, M. (1997). Antecedents of dental anxiety: Learned responses versus personality traits. Community Dentistry and Oral Epidemiology, 25, 233-237.

Giron, M. C. C. (1988). Fundamentos psicológicos da prática odontológica. Porto Alegre: D. C. Luzzatto.
Graminha, S. S. V. (1994). A escala comportamental infantil de Rutter A2: estudos de adaptação e fidedignidade. Estudos de Psicologia, 11(3), 34-42.

IBGE - Instituto Brasileiro de Geografia e Estatística (2000). Resultados da amostra do censo demográfico 2000. Recuperado em 15 de Fevereiro de 2006 de http://www.ibge.gov.br.

Jacob, L. S. (1998). Psicologia e odontologia. Em B. Rangé (Org.), Psicoterapia Comportamental e Cognitiva. São Paulo: Editorial Psy.

Klatchoian, D. A. (1993). Psicologia odontopediátrica. São Paulo: SARVIER.

Lipp, M. E. N. (2000). Inventário de sintomas de stress para adultos de Lipp (ISSL): manual. São Paulo: Casa do Psicólogo.

Lipp, M. E. N. \& Lucarelli, M. D. M. (1998). Escala de stress infantil - ESI: manual. São Paulo: Casa do Psicólogo.

Lipp, M. E. N., Souza, E. A. P., Romano, A. S. P. F. \& Covolan, M. A. (1991). Como enfrentar o stress infantil. São Paulo: Ícone.

Macchi, R., Biondi, A. M. \& Cortese, S. G. (1996). Influencias de variables en la calidad del desempeño clínico en alumnos de la cátedra de odontología integral niños. Revista de la Facultad Odontología, 16(41), 35-40.

Moraes, A. B. A., Ambrosano, G. M. V. \& Possobon, R. de F. (2004). Fear assessment in Brazilian children: The relevance of dental fear. Psicologia: Teoria e Pesquisa, 20(3), 289-294.

Newton, J. T., Baghaienaini, F., Goodwin, S. R., Invest, J., Lubbock, M. \& Marouf Saghakhaneh, N. (1994). Stress in dental school: A survey of students. Dent Update, 21, 162-164.

Possobon, R. de F., Caetano, M. E. S. \& Moraes, A. B. A. (1998). Odontologia para crianças não colaboradoras: relato de casos. Revista Brasileira de Odontologia, 55(2), 80-83.

Selye, H. A. (1956). The stress of life. New York: Longmans.

Selye, H. A. (1983).The stress concept: Past, present, and future. In C. L. Cooper (Ed.), Stress research: Issues for the eighties (pp.1-20). New York: John Wiley \& Sons.

Nie, N. H., Hull, C. H. \& Bent, D. H. (1968). Statistic package for social sciences - SPSS (Version 12.0 for Windows $\AA$ ) [Programa estatístico para computador]. Chicago: SPSS. (ISBN 0-13-654352-9 No 60611).

Sturdevant, J. R., George, J. M. \& Lundeen, T. F. (1987). An interactional view of dental student stress. Journal of Dental Education, 51(5), 246-49.

Tedesco, L. A. (1986). A psychosocial perspective on the dental educational experience and student performance. Journal of Dental Education, 50, 601-5.

Recebido em 31/03/06 Aceito em 14/06/2006

Endereço para correspondência: Cármen Lúcia Cardoso. Faculdade de Filosofia Ciências e Letras de Ribeirão Preto - USP, Departamento de Psicologia e Educação. Av. Bandeirantes, 3900, CEP 14040-901, Ribeirão Preto-SP. E-mail: carmen@ffclrp.usp.br 